\title{
Vulnerabilidade à contaminação das águas superficiais da Bacia Hidrográfica do Rio Pirapó, Paraná
}

\section{Vulnerability to surface water contamination of Pirapó River Basin, Paraná}

\author{
Carlos Henrique da Graça* $\bowtie(\mathbb{D}$, Hélio Silveira $\bowtie(\mathbb{D}$ \\ Departamento de Geografia, Universidade Estadual de Maringá, Maringá, Paraná, Brasil \\ E-mail: hesilveira70@hotmail.com \\ *E-mail para correspondência: henriquechg@gmail.com
}

Recebido (Received): 26/09/2019

Resumo: A água é um dos recursos mais importantes para a sobrevivência dos seres vivos, devendo ser muito bem gerida, de modo a não comprometer a qualidade de vida das gerações futuras. Um dos maiores desafios é diminuir os efeitos da carência e contaminação da água. O objetivo desse trabalho é estabelecer uma classificação da vulnerabilidade à contaminação das águas superficiais, visando contribuir com a gestão mais efetiva da bacia hidrográfica do rio Pirapó - Paraná. Para isso foi aplicado o modelo semi-empírico de Morgan, Morgan e Fenney (MMF - modificado). Os resultados mostram que vulnerabilidade à contaminação das águas superficiais apresentou maior porcentagem ligada à vulnerabilidade média, corresponde cerca de $63 \%$ da área da bacia. Essa classificação, está relacionada a ocorrência de solos profundos (Latossolos e Nitossolos) e resistentes ao desprendimento de partículas, com valores mais baixos de escoamento superficial influenciados pela redução na intensidade da precipitação, além de estar sobre áreas de declividade moderadas, com predomino de classes ente 6 e $15 \%$. Assim, considera-se que o estudo possa contribuir no desenvolvimento ações favoráveis a proteção das águas superficiais da bacia, priorizando áreas específicas de proteção para o monitoramento de suas águas.

Palavras-chave: Vulnerabilidade à contaminação; Escoamento superficial; Agroquímicos; Modelo MMF.

Abstract: Water is one of the most important resources for the survival of living beings, and must be very well managed, so as not to compromise the quality of life of future generations. One of the biggest challenges is to reduce the effects of water deficiency and contamination. The objective of this work is to establish a classification of the vulnerability to the contamination of surface waters, aiming to contribute with the more effective management of the hydrographic basin of the Pirapó - Paraná River. For this, a semi-empirical model of Morgan, Morgan and Fenney (MMF - modified) was applied. The results show that vulnerability to surface water contamination showed a higher percentage linked to the average vulnerability, corresponding to approximately $63 \%$ of the basin area. This classification is related to the occurrence of deep soils (Oxisols and Nitosols) and resistant to the release of particles, with lower values of surface runoff influenced by the reduction in the intensity of precipitation, in addition to being over moderate slope areas, with a predominance of classes between 6 and $15 \%$. Thus, it is considered that the study may contribute to the development of more favorable actions for the protection of surface waters in the basin, prioritizing specific areas of protection for the monitoring of its waters.

Keywords: Vulnerability to contamination; Runoff; Agrochemicals; MMF model.

\section{Introdução}

A promoção de qualquer forma de desenvolvimento em uma região leva a sociedade (rural ou urbana) a causar impactos negativos no ambiente em que vivem, podendo ser percebidos pelo mau uso do solo e da água. Processos como erosão, lixiviação e modificação da cobertura vegetal ocorrem de forma natural, mas quando o homem altera o ambiente, esses processos são acentuados, levando a consequências imediatas de usos inadequados do solo (SILVA et al., 2007). Boa parte da quantidade e da qualidade da água é afetada 
pelo uso indiscriminado do solo e pela concentração de nutrientes, esgotos e produtos tóxicos em função do crescimento desordenado de áreas urbanas, industriais e agrícolas (SPERLING, 2007).

O uso e manejo dos solos são indicadores de grande importância para gestão dos recursos hídricos e a saúde de uma bacia hidrográfica, uma vez que a qualidade dos corpos d'água é função das ações que ocorrem ao longo das suas vertentes. A quantidade de contaminantes físico-químicos e biológicos em águas superficiais, por exemplo, aumenta diretamente com a presença humana e com a agricultura intensiva (SUTTON; FISHER, 2009). Por sua vez, a qualidade da água reduz à medida que resíduos e agroquímicos são dispostos nas vertentes (OLLI et al., 2009).

O conhecimento do comportamento das propriedades dos solos e os efeitos impactantes causados pelo tipo de manejo, colaboram para maior conhecimento e precisão dos potenciais de escoamento e infiltração em bacias hidrográficas, por unidade de solo, posterior identificação e delimitação de áreas de risco de contaminação, tanto de águas superficiais quanto de águas subsuperficiais (SPADOTTO et al., 2012).

Em meio a essa preocupação, são desenvolvidos constantemente modelos para avaliar a vulnerabilidade dos corpos d'água a contaminação (SPADOTTO et al., 2012). No entanto, para que esses modelos possam ser utilizados na avaliação de áreas específicas, é necessário levar em consideração as características de elemento contaminante, o que traz complexidade a aplicação, além demandarem diversos dados físicos de entrada (MINGOTI et al., 2016). Para Tesfahunegn et al. (2014) a aplicação de modelos baseados em processos físicos nem sempre é uma tarefa fácil, pois exige grandes quantidades de informações que geralmente não estão disponíveis, principalmente nas regiões em desenvolvimento com escassez de dados.

O modelo MMF - modificado (MORGAN, 2001) foi selecionado como base para estudo devido à sua simplicidade e flexibilidade de aplicação (LILHARE et al., 2015; DEVIA et al. 2015), sendo um modelo semi-empírico com base física que demanda menos dados de entrada do que a maioria dos outros modelos preditivos de escoamento superficial e o fluxo de sedimentos (EFTHIMIOU et al., 2017; EFTHIMIOU, 2019), que serviram de base estimar a vulnerabilidade a contaminação, devido a capacidade de arraste de sedimentos e, consequentemente, a deposição de contaminantes nos rios.

A escolha da bacia hidrográfica do rio Pirapó para a pesquisa se deu em virtude da grande importância que possui dentro do contexto agropecuário do norte-central paranaense, apresentando setor primário com grande representatividade na economia dos municípios que a compõe, juntamente com o fato de que suas águas serem fontes de abastecimento de mais de um milhão de habitantes e receptora de efluentes de origens variadas. Assim, justifica-se a necessidade do presente trabalho destacar a bacia, pois se infere que esteja ocorrendo sistematicamente alterações no meio natural e, portanto, à contaminação das águas superficiais da bacia (FREIRE , 2010, SILVA, 2017), proporcionada pela expansão das áreas urbanas e agrícolas, com consequente intensificação do despejo de efluentes, da demanda de insumos agrícolas e da formação de processos erosivos (ALVES, 2008; FREIRE et al., 2014). Desse modo, objetiva-se nesse trabalho estabelecer uma classificação da vulnerabilidade à contaminação das águas superficiais, visando contribuir com a gestão mais efetiva da bacia hidrográfica do rio Pirapó - Paraná.

\section{Caracterização da área de estudo}

A bacia do rio Pirapó está localizada na porção norte central do Estado do Paraná, ocupando uma área de $5.098 \mathrm{Km}^{2}$ (Figura 1), abrangendo total ou parcialmente 33 municípios, dentre os quais nove municípios utilizam abastecimento por água superficial para o consumo humano nas cidades.

O clima predominante na região da bacia é subtropical úmido mesotérmico (Cfa), com verões quentes e geadas pouco frequentes durante o outono e inverno, com tendência de concentração de chuvas nos meses de verão. As temperaturas médias anuais variam entre $21^{\circ} \mathrm{C}$ e $24^{\circ} \mathrm{C}$ e valores pluviométricos médios anuais entre 1.200 a $1.600 \mathrm{~mm}$ (NITSCHE et al., 2019).

O substrato geológico da bacia caracterizada pela presença de uma zona de transição entre diferentes unidades litológicas, constituído por rochas basálticas da Formação Serra Geral, os arenitos das Formações Caiuá; Santo Anastácio e Adamantina e sedimentos recentes (MINEROPAR, 2001).

O relevo da bacia apresenta uma diferença altimétrica de 608 metros, com altitudes variando entre 264 metros, próximas a calha do rio Paranapanema, a 872 metros no seu limite superior extremo. O comportamento do relevo apresenta uma maior monotonia nas suas formas, com predominância de declividades inferiores a 8\%. Entretanto, a montante o relevo se faz mais dissecado com presença de vales entalhados e declividades mais acentuadas, superiores a 20\% (NÓBREGA et al.,2015). 


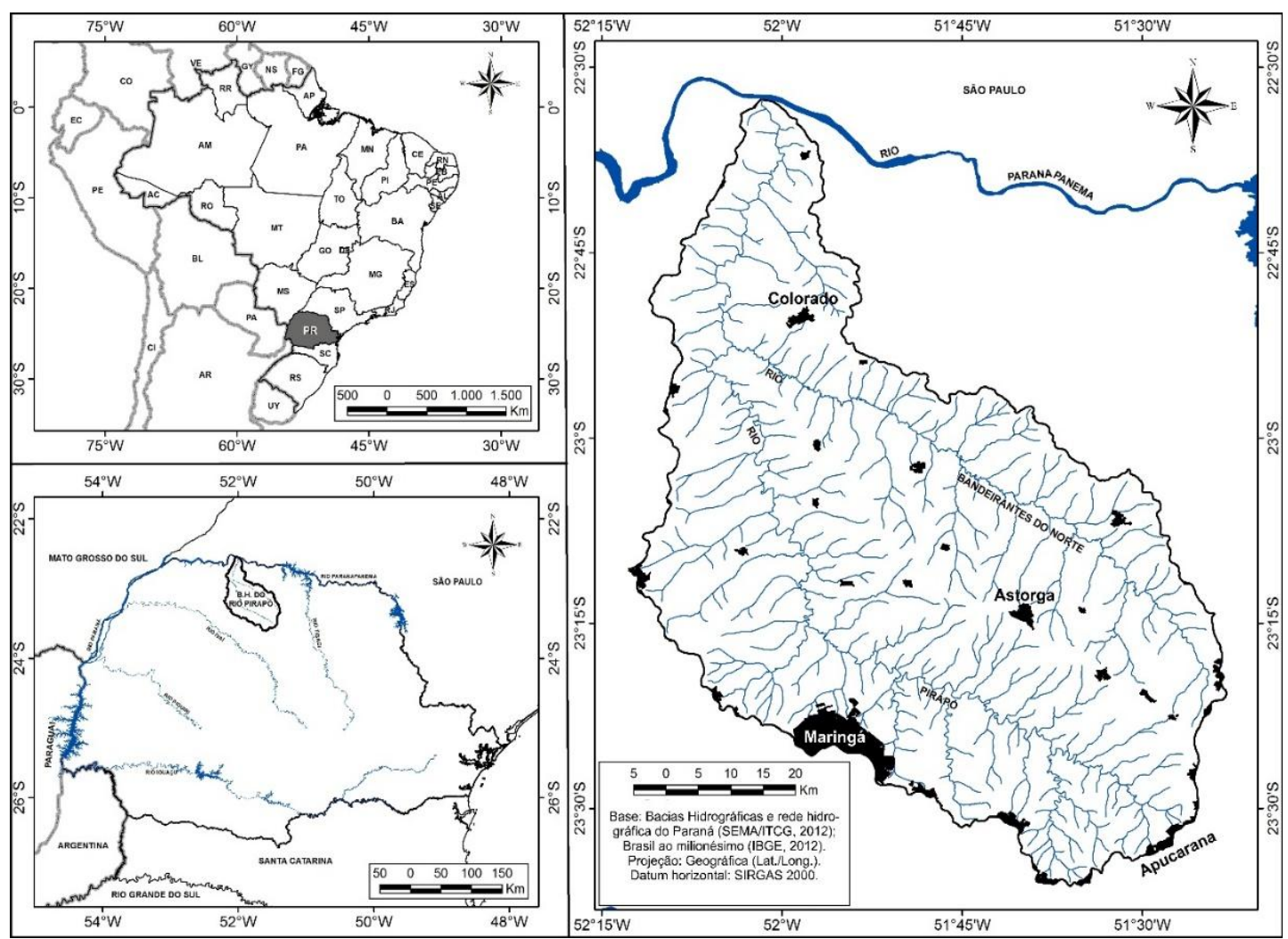

Figura 1: Localização da bacia hidrográfica do Rio Pirapó.

Nos setores onde os solos são oriundos da alteração de rochas básicas ocorrem os Latossolos Vermelhos, sobre declividades entre 6 a 12\%; Nitossolos Vermelhos, em declividades entre 6 a 20\%, ambos com textura argilosa a muito argilosa; e Neossolos Litólicos e Regolíticos, em declividades superiores a $20 \%$. Na região de ocorrência das rochas areníticas, predominam os Latossolos Vermelhos de textura média, sobre declividades inferiores a 8\%; e os Argissolos Vermelhos de textura arenosa/média, em declividades superiores a 6\% (Figura 2 - A) (EMBRAPA, 2007).



Figura 2: A) Classes de solos e B) Cobertura vegetal e uso da terra da bacia do rio Pirapó - PR. 
A cobertura vegetal e uso da terra da bacia apresenta predominantemente culturas extensivas, como cultivos temporárias (cultivo de soja, milho e trigo) e a cana-de-açúcar, que ocupam juntos aproximadamente $70 \%$ da área total da bacia, onde ocorrem sobre variadas classes pedológicas e, principalmente em relevo com baixas declividades e vertentes alongadas (NÓBREGA et al., 2015). As pastagens ocupam cerca de $23 \%$ da área total da bacia, concentrando-se nas áreas de relevo mais dissecado, no alto vale do Pirapó, com predomínio de solos rasos e, em partes do setor médio, nas áreas de solos de textura média/arenosa, diminuindo a sua representatividade em direção ao norte da bacia, baixo vale do Pirapó, onde vem sendo substituída sistematicamente pelo cultivo da cana-de-açúcar. Todos os outros usos somados ocupam uma área de aproximadamente $9,6 \%$ da bacia, sendo representados pelas áreas urbanas, cultivos permanentes e floresta/matas (Figura 2 - B).

\section{Materiais e métodos}

A vulnerabilidade à contaminação das águas superficiais foi proposta para a bacia baseando-se nos resultados obtidos com a aplicação do modelo semi-empírico de Morgan, Morgan e Finney (1984 - MMF) revisado por Morgan (2001), com algumas modificações propostas por Vigiak et al. (2005) e Luger et al. (2018).

A energia cinética da precipitação é dada em função da precipitação efetiva anual (ER, eq. 1), baseada no fator de intercepção (1-A), que representa a proporção da precipitação permanentemente interceptada pelo tipo de cobertura do solo. Os valores típicos de $A$ para os diferentes tipos de cobertura do solo foram atribuídos com base em Morgan (1995) e Vigiak et al. (2005) (Tabela 1).

$$
\mathrm{ER}=\mathrm{R} *(1-A)
$$

Onde: ER - precipitação efetiva anual, $\mathrm{mm} ; \mathrm{R}$ - precipitação média anual, $\mathrm{mm} ; A$ - proporção (entre 0 - 1 ) da precipitação interceptada pela cobertura do solo.

Tabela 1: Dados de entrada sobre cobertura vegetal e uso da terra da bacia do rio Pirapó - PR.

\begin{tabular}{lcccc}
\hline \multicolumn{1}{c}{ Cobertura vegetal e uso da terra } & $\mathbf{( 1 - A )}$ & CC & PH (m) & GC \\
\hline Vegetação densa/Matas & 0,30 & 0,67 & 3,93 & 0,89 \\
Cultivos permanentes (café e laranja) & 0,30 & 0,52 & 1,50 & 0,77 \\
Cultivos temporários (soja, milho e trigo) & 0,17 & 0,30 & 0,67 & 0,49 \\
Cultivos de cana-de-açúcar & 0,25 & 0,37 & 0,91 & 0,55 \\
Pastagem & 0,30 & 0,20 & 0,08 & 0,60 \\
Silvicultura & 0,28 & 0,30 & 8,00 & 0,73 \\
\hline
\end{tabular}

Fonte: Brandt (1990), Morgan (1995) e Vigiak et al. (2005).

A precipitação efetiva anual (ER) foi dividida entre a que atinge a superfície do solo como passagem direta (DT - eq. 2) e aquela que é interceptada pelo dossel da planta e que atinge a superfície por escoamento de haste ou gotejamento das folhas anual (LD - eq. 3). A divisão é uma função direta da porcentagem de cobertura do dossel $\left(\mathrm{CC}, \mathrm{m}^{2} \cdot \mathrm{m}^{-2}\right)$ (Tabela 1).

$$
\begin{aligned}
& \mathrm{LD}=\mathrm{ER} * \mathrm{CC} \\
& \mathrm{DT}=\mathrm{ER}-\mathrm{LD}
\end{aligned}
$$

Onde: $\mathrm{LD}$ - precipitação efetiva com passagem direta anual, $\mathrm{mm}$; ER - precipitação efetiva anual, $\mathrm{mm}$; CC Porcentagem de cobertura do dossel (entre 0 e 1) segundo Morgan (2001) e Vigiak et al. (2006); DT precipitação efetiva interceptada pelo dossel da planta, $\mathrm{mm}$.

A energia cinética total da chuva anual (KE, MJ. ha ${ }^{-1}$ ) é dada pela soma da energia cinética da precipitação direta anual $\left(\mathrm{KE}_{\mathrm{DT}}\right.$ - eq. 4$)$, que é função da intensidade da chuva erosiva $\left(I, \mathrm{~mm} \cdot \mathrm{h}^{-1}\right)$, e da energia cinética por escoamento de haste ou gotejamento das folhas $\left(\mathrm{KE}_{\mathrm{LD}}-\right.$ eq. 5), que é função da altura da vegetação (PH), como proposto por Brandt (1990) (Tabela 1). Os valores de correção linear e angular utilizados na eq. 4 foram propostos por Rufino et al. (1993), assumindo padrões de erosividade da chuva da região 4 do estado de Paraná que compreende a área da bacia (TERASSI et al., 2016). Quando a eq. 5 produz valores negativos, a energia de drenagem das folhas assume valor igual a zero (MORGAN, 2001). 


$$
\begin{array}{r}
\mathrm{KE}_{\mathrm{DT}}=\mathrm{DT}^{*}(0,1673+0,0402 \log I) \\
\mathrm{KE}_{\mathrm{LD}}=\left(0,158 * \mathrm{PH}^{0.5}\right)-0,587
\end{array}
$$

Onde: $\mathrm{KE}_{\mathrm{DT}}$ - energia cinética com passagem direta anual, MJ.ha ${ }^{-1}$; DT - precipitação efetiva interceptada pelo dossel da planta anual, $\mathrm{mm} ; I$ - intensidade de chuva erosiva, foi adotado o valor típico de $25 \mathrm{~mm} . \mathrm{h}^{-1}$ relacionado aos climas tropicais (HUDSON, 1965 apud VIGIAK et al., 2005); $\mathrm{KE}_{\mathrm{LD}}$ - energia cinética por escoamento de haste ou gotejamento das folhas anual, $\mathrm{MJ} \cdot \mathrm{ha}^{-1} \cdot \mathrm{ano}^{-1} ; \mathrm{PH}$ - altura da vegetação, $\mathrm{m}$.

O escoamento superficial foi classificado seguindo o modelo adotado de Agnindhira (2016) e Luger et al. (2018), (eq. 6), estabelecido pela multiplicação da diferença entre a precipitação (P) e a evapotranspiração real (ETR) anuais, com o coeficiente de escoamento (C).

$$
\mathrm{Q}_{\text {anual }}=(\mathrm{P}-\mathrm{ETR}) * \mathrm{C}
$$

Onde: $Q_{\text {anual }}$ - escoamento superficial anual, mm.ha ${ }^{-1} ; \mathrm{P}$ - Precipitação anual, mm; ETR - Evapotranspiração real anual, $\mathrm{mm} ; \mathrm{C}$ - coeficiente de escoamento, adimensional.

O coeficiente de escoamento foi calculado a partir da multiplicação dos fatores de proteção por tipo de uso e manejo do solo (c e p - Tabela 2) (VIGIAK et al. 2005) da Equação Universal de Perda de Solo (WISCHMEIER; SMITH, 1978), com os ângulos de declive do terreno (S, graus), baseado nas recomendações do Soil Conservation Service (SCS - USDA) (PRUSKI et al., 2008).

Tabela 2: Índices de c e p atribuídos a cobertura vegetal e uso da terra na bacia do rio Pirapó - PR.

\begin{tabular}{lcc}
\hline \multicolumn{1}{c}{ Cobertura vegetal e uso da terra } & Fator (c) & Fator (p) \\
\hline Vegetação densa/Matas & $0,001^{1}$ & $0,1^{1}$ \\
Cultivos permanentes (café e laranja) & $0,135^{2}$ & $0,5^{1}$ \\
Cultivos temporários (cana-de-açúcar, soja, milho e trigo) & $0,5^{1}$ & $0,5^{1}$ \\
Pastagem & $0,01^{1}$ & $0,4^{1}$ \\
Silvicultura (Eucalipto) & $0,04892^{3}$ & $0,21^{1}$ \\
\hline
\end{tabular}

Fonte: Fator c - ${ }^{1}$ Tomazoni e Guimarães (2005); ${ }^{2}$ Vázquez-Fernández et al. (1996) e ${ }^{3}$ Oliveira et al. (2007); Fator p - ${ }^{1}$ Bertoni e Lombardi Neto (2012).

Os valores aplicados ao uso e manejo do solo (fator c) foram estabelecidos a partir dos trabalhos de Vázquez-Fernández et al. (1996) e Tomazoni e Guimarães (2005). A atribuição dos valores da prática conservacionista (fator p) seguiu os critérios de Bertoni e Lombardi Neto (2012), sendo o plantio em contorno a principal prática adota na bacia (Tabela 2).

Os dados pluviométricos foram obtidos em 12 postos pluviométricos do Instituto das Águas do Paraná e em estações meteorológicas da rede do IAPAR/SIMEPAR, no município de Apucarana, e INMET, no município de Maringá, distribuídos no interior e no entorno da bacia (TERASSI et al., 2016), com série temporal compreendida entre 1976 a 2016.

Os valores de temperatura média anual do ar foram estimados aplicando os coeficientes de regressão linear das equações mensais e anual propostos por Pinto e Alfonsi (1974) (eq. 8), que leva em consideração as informações de latitude e altitude do ponto a ser considerado. Na bacia, utilizou-se as localizações dos postos pluviométricos como referência para os cálculos. Foram utilizados para medir o nível de correlação linear os dados médios mensais de temperatura do ar referentes ao período compreendido entre 1976 a 2016 das estações meteorológicas de Apucarana e Maringá.

$$
\mathrm{Y}=\mathrm{a}+\mathrm{b} \cdot \mathrm{x}_{1}+\mathrm{c} \cdot \mathrm{x}_{2}
$$

Onde: $\mathrm{Y}$ - estimativa da temperatura $\left({ }^{\circ} \mathrm{C}\right) ; \mathrm{x}_{1}$ - altitude $(\mathrm{m}) ; \mathrm{x}_{2}$ - latitude (minutos); $\mathrm{a}, \mathrm{b}$ e c - coeficiente de regressão das equações mensais e/ou anual.

A evapotranspiração real (ETR) anual foi estimada por meio do método proposto por Turc (1954), desenvolvido no Instituto Nacional de Pesquisas Agronômicas Francês a partir da relação entre precipitação e temperatura média anual (eq. 9 e 10). 


$$
\begin{gathered}
\mathrm{ETR}=\frac{\mathrm{P}}{\sqrt{0,9+\frac{\mathrm{P}^{2}}{\mathrm{~L}^{2}}}} \\
\mathrm{~L}=300+25 \mathrm{~T}+0,05 \mathrm{~T}^{3}
\end{gathered}
$$

Onde: ETR - Evapotranspiração real média anual, mm; P - Precipitação média anual, mm; L -Parâmetro empírico dado pela eq. $10 ; \mathrm{T}$ - Temperatura média anual, ${ }^{\circ} \mathrm{C}$.

Os valores de energia cinética derivados da fase aquosa são utilizados no modelo para estimar o destacamento de partículas do solo pelo impacto das gostas de chuva $(\mathrm{F}-$ eq. 11$)$ e pelo escoamento $(\mathrm{H}-$ eq. 12) aplicando uma simplificação das equações de erosão descrita por Meyer e Wischmeier (1969 apud LUGER et al., 2018).

$\mathrm{O}$ primeiro depende da capacidade de separação do solo (erodibilidade, $\mathrm{K}$ - eq. 13), enquanto que o segundo depende da coesão do solo $(\mathrm{COH})$. O destacamento do solo devido o escoamento e a capacidade de transporte dependem ainda do volume do escoamento superficial $(\mathrm{Q})$, dos fatores de cobertura $(\mathrm{GC}$ e $\mathrm{C})$ e do declive (S).

$$
\begin{array}{r}
F=K^{*} \mathrm{KE}^{*} 10^{-3} \\
\mathrm{H}=\mathrm{ZQ}^{1,5} \sin \mathrm{S}(1-\mathrm{GC}) * 10^{-3}
\end{array}
$$

Onde: F - destacamento de partículas do solo pelo impacto das gostas de chuva, t.ha ${ }^{-1} \cdot \mathrm{ano}^{-1}$, K -


de partículas do solo por escoamento superficial, t.ha ${ }^{-1}$.ano ${ }^{-1} ; \mathrm{Z}$ - resistência do solo; Q - escoamento superficial, mm.ha ${ }^{-1}$.ano ${ }^{-1} ; \mathrm{S}$ - ângulo de declive do terreno, graus; GC - porcentagem de cobertura do solo (entre 0 e 1), valores típicos extraídos de Vigiak et al. (2005) (Tabela 1).

Os valores de $\mathrm{K}$, correspondentes à erodibilidade dos solos, foram estimados pelo método proposto por Bouyoucos (1935) utilizando dados da composição granulométrica dos solos da bacia apresentados por Marcatto (2016) e Marcatto e Silveira (2017), para profundidades de até 0,4 metros sobre diferentes tipos de cobertura e usos da terra (Tabela 3).

Para a aplicação na eq. 12 os valores de coesão da superfície do solo $(\mathrm{COH}, \mathrm{kPa})$ são convertidos em resistência do solo (Z, eq. 13) baseado na simplificação do trabalho de Rauws e Govers (1988 apud MORGAN, 2001). Para solos soltos e não coesos, $Z=1,0$. Devido à falta de dados sobre a coesão dos solos na bacia os valores típicos utilizados foram extraídos de Morgan et al. (1993) (Tabela 3). A identificação dos valores foi realizada a partir das classes texturais dos solos (MARCATTO, 2016; MARCATTO;

SILVEIRA, 2017).

$$
\mathrm{Z}=\frac{1}{(0.5 \mathrm{COH})}
$$

A capacidade de transporte do escoamento superficial (TC, eq. 14) foi definida como a concentração máxima de sedimentos que pode ser transportado (GOVERS, 1990).

$$
\mathrm{TC}=C Q^{2} \sin S^{*} 10^{-3}
$$

Onde: TC - capacidade de transporte do escoamento anual, t.ha ${ }^{-1}$; C - coeficiente de escoamento, eq. 6; S ângulo de declive, graus. 
Tabela 3: Dados de entrada sobre cobertura vegetal e uso da terra da bacia do rio Pirapó - PR.

\begin{tabular}{|c|c|c|c|c|c|c|c|c|}
\hline \multirow[b]{2}{*}{ Solos } & \multirow{2}{*}{$\begin{array}{l}\text { Tipos de } \\
\text { usos }\end{array}$} & \multirow{2}{*}{$\frac{\text { Prof. }}{(\mathbf{c m})}$} & \multicolumn{3}{|c|}{ Granulometria (\%) } & \multirow{2}{*}{$\begin{array}{c}\text { M.O. } \\
(\text { g.dm } \\
\text { (-3) }\end{array}$} & \multirow{2}{*}{$\frac{\text { K - erodibilidade }}{\left(\text { g.J }^{-1}\right)}$} & \multirow{2}{*}{$\frac{\mathrm{COH}}{(\mathrm{kPa})}$} \\
\hline & & & Areia & Silte & Argila & & & \\
\hline \multirow{3}{*}{ ב } & (p) & & 5,04 & 26,2 & 68,76 & 40,63 & 0,0454 & \multirow{3}{*}{12} \\
\hline & (c) & $0-40$ & 8,995 & 21,855 & 69,15 & 26,53 & 0,0446 & \\
\hline & (f) & & 5,815 & 30,32 & 63,865 & 39,63 & 0,0566 & \\
\hline \multirow{4}{*}{ 之 } & (p) & & 10,045 & 25,27 & 64,685 & 42,29 & 0,0546 & \multirow{3}{*}{12} \\
\hline & (c) & $0-40$ & 8,595 & 19,27 & 72,135 & 30,56 & 0,0386 & \\
\hline & (f) & & 6,57 & 23,97 & 69,46 & 29,89 & 0,0440 & \\
\hline & (p) & & 84,875 & 2,535 & 12,59 & 7,39 & 0,6943 & \multirow{4}{*}{2} \\
\hline \multirow{4}{*}{ ב } & (c) & & 82,44 & 2,6 & 14,96 & 7,06 & 0,5684 & \\
\hline & (ca) & $0-40$ & 74,51 & 3,43 & 22,06 & 13,09 & 0,3533 & \\
\hline & (f) & & 80,5 & 2,615 & 16,885 & 29,89 & 0,4922 & \\
\hline & (p) & & 78,565 & 9,14 & 12,295 & 17,46 & 0,7133 & \multirow{4}{*}{2} \\
\hline \multirow{3}{*}{ 文 } & (c) & & 87,195 & 2,98 & 9,825 & 9,07 & 0,9178 & \\
\hline & (ca) & $0-40$ & 85,93 & 2,935 & 11,135 & 6,71 & 0,7981 & \\
\hline & (f) & & 83,46 & 3,765 & 12,775 & 18,14 & 0,6828 & \\
\hline \multirow{3}{*}{ 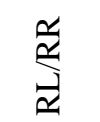 } & (p) & $0-19$ & 24,8 & 47 & 28,2 & 42,98 & 0,2546 & \multirow{3}{*}{3} \\
\hline & (c) & $0-16$ & 9,4 & 67,9 & 22,7 & 32,91 & 0,3405 & \\
\hline & (f) & $5-30$ & 5,6 & 62,1 & 32,3 & 66,49 & 0,2096 & \\
\hline
\end{tabular}

LV - Latossolo Vermelho (text. argilosa); NV - Nitossolo Vermelho (text. argilosa); LVm - Latossolo Vermelho (text. média); PV Argissolo Vermelho (text. arenoso); RL/RR - Neossolo Litólico/Regolítico (text. franco-argilosa); p - pastagem; c - cultivos temporários; ca - cana-de-açúcar; f - floresta; M.O. - Matéria orgânica. Fonte: Morgan et al. (1993); Marcatto (2016) e Marcatto e Silveira (2017).

A taxa de desprendimento total anual dos solos (TD, t.ha ${ }^{-1}$ ) foi estimada pelo somatório entre o destacamento das partículas do solo pelo impacto da gota de chuva $(\mathrm{F})$ e o destacamento das partículas do solo pelo escoamento superficial $(\mathrm{H})$ anual. $\mathrm{O}$ impacto das gotas de chuva aumenta a capacidade de transporte do fluxo e, através do destacamento das partículas do solo, provoca concentrações mais altas de sedimentos.

A classificação da vulnerabilidade à contaminação das águas superficiais foi realizada em função da relação da capacidade de transporte do escoamento superficial com a taxa anual de desprendimento de partículas do solo, uma vez que, a água representa a principal forma de dispersão de contaminantes no ambiente (SPADOTTO et al., 2012). Assim, quanto maior as partículas desprendidas do solo, maior a chance de transporte dessas partículas junto com contaminantes. O produto dessa relação foi associado com a possibilidade de carreamento de agroquímicos por fluxo superficial, perfazendo uma relação de intensificação da vulnerabilidade à contaminação.

Para cada tipo de uso identificado na bacia foi atribuído um valor de potencialização (entre 0 e 1) de acordo com o consumo de agroquímicos em cada cultura (Tabela 4), baseado no Plano de Vigilância e Atenção à Saúde de Populações Expostas aos Agroquímicos do Estado do Paraná 2017 a 2019 (SESA, 2018). No estado do Paraná, os maiores consumidores de agroquímicos são os cultivos temporários (soja, milho, trigo e cana) responsáveis por cerca de $80 \%$ do total, seguido pela pastagem com $2,23 \%$ e os cultivos permanentes (citros e café) com cerca de 2\% (SIAGRO, 2018).

Os valores atribuídos foram definidos de acordo com Alves Filho (2002), que apresenta um estudo de que apenas 10\% dos agroquímicos aplicados diretamente nas plantas ou no solo atingem efetivamente seu alvo, o restante tem como destino final o solo, sendo lavados das folhas através da ação da chuva ou da água de irrigação (SCORZA JUNIOR et. al., 2010). Assim, assume-se que todos os cultivos identificados na bacia intensificam em $90 \%$ o risco de contaminação por área com agroquímicos (eq. 15; Tabela 4).

$$
\mathrm{V}_{\mathrm{as}}=\frac{\mathrm{TC}}{\mathrm{Tx}}+\exp ^{\left(\mathrm{C}_{\mathrm{iv}}\right)}
$$

Onde: $\mathrm{V}_{\mathrm{as}}$ - vulnerabilidade à contaminação das águas superficiais; TC - Capacidade de transporte pelo escoamento superficial anual, t.ha ${ }^{-1}$; TD - taxa de desprendimento total anual dos solos, t.ha ${ }^{-1}$; $\mathrm{C}_{\mathrm{iv}}-$ coeficiente de intensificação da vulnerabilidade à contaminação (agroquímicos). 
Tabela 4: Valores de coeficiente de intensificação da vulnerabilidade à contaminação (agrotóxico) por classes de cobertura vegetal e uso da terra da bacia do rio Pirapó - PR.

\begin{tabular}{lccc}
\hline \multicolumn{1}{c}{ Cobertura vegetal e uso da terra } & \% US & $\mathbf{\%}$ FIR & $\mathbf{C}_{\mathbf{i v}}$ \\
\hline Vegetação densa/Matas e Silvicultura (Eucalipto) & 0,0 & 0,0 & 0,0 \\
Cultivos permanentes (café e laranja) & $2,0 \%$ & $90 \%$ & 0,02 \\
Cultivos temporários (cana-de-açúcar, soja, milho e trigo) & $80 \%$ & $90 \%$ & 0,9 \\
Pastagem & $2,23 \%$ & $90 \%$ & 0,024 \\
\hline
\end{tabular}

Proposto pelos autores.

Os valores de coeficiente de intensificação da vulnerabilidade à contaminação foram definidos estabelecendo uma relação entre a porcentagem de usos de agroquímicos (\% US) por cultivo no Estado e porcentagem do fator que intensificam o risco de contaminação (\% FIR) (Tabela 4). Para as áreas com vegetação densa/matas e silvicultura adotou-se o valor zero (0), devido ao não registro de uso de agroquímicos. A vulnerabilidade à contaminação das águas superficiais foi classificada seguindo uma divisão simples dos valores correspondentes entre zero (0) e seis (6) (Tabela 5).

Tabela 5: Intervalos de classes da vulnerabilidade à contaminação das águas superficiais

\begin{tabular}{cc}
\hline Classes $\mathbf{V}_{\text {as }}$ & Intervalos \\
\hline Muito Elevada & $4,8-6,0$ \\
Elevado & $3,6-4,8$ \\
Média & $2,4-3,6$ \\
Baixa & $1,2-2,4$ \\
Muito baixa & $0,0-1,2$ \\
\hline
\end{tabular}

Proposto pelos autores.

\subsection{Elaboração dos produtos cartográficos}

O material cartográfico foi gerado e editado utilizando software Arcgis (ArcMap) 10.4.1 free trial, com base sistema de projeção Geográfica (Lat./Long.) e Datum horizontal SIRGAS 2000. Os produtos da aplicação dos métodos, modelo MMF - modificado, que resultaram na classificação da vulnerabilidade a contaminação das águas superficiais foram gerados a partir dos arquivos vetoriais das classes de solos, tipos de usos do solo e dos rasters de pluviosidade, temperatura do ar, evapotranspiração (ETR) e declividade. Para espacialização da pluviosidade, temperatura do ar e evapotranspiração da bacia utilizou o interpolador Kriging (Ordinary-spherical) (SILVA et al., 2010).

Os tipos de uso do solo na bacia foram definidos por classificação supervisionada (MAXVER) em imagens de satélite LandSat 8, sensor OLI (órbitas 222 e 223 e ponto 76 de 23/10 e 01/11 de 2016), com resolução espacial de 30 metros, obtidas via sítio eletrônico do U.S Geological Survey (USGS-GLOVIS), bem como, em trabalhos de campo para coleta dos pontos de amostragens visando minimizar erros de interpretação visual das classes estabelecidas durante a rotina de processamento.

Para atribuição dos valores considerados em cada variável descrita na metodologia foi necessária a reclassificação dos arquivos bases. Posteriormente os planos de informações gerados foram direcionados a ferramenta Raster Calculator (ArctoolBox - Spacial Analyst Tools - Map Algebra), onde foi realizado todos os cálculos descritos na metodologia.

\section{Resultados e discussões}

A precipitação efetiva apresentou uma média anual de $1.160 \mathrm{~mm}$, correspondendo a $79 \%$ da precipitação total interceptada pela vegetação, com valores entre 1.418 a $860 \mathrm{~mm}$ e maior intensidade no setor centro-sul da bacia (Figura 3 - A), que representa os maiores acumulados de precipitação total e intenso uso agrícola do solo. A precipitação efetiva, na agricultura, é uma parte da precipitação total, que infiltra no solo, mas que fica nas camadas mais superficiais. O restante da água é perdido por escoamento superficial e por drenagem profunda (STEENHUIS et al., 1995). A cobertura vegetal além de reduzir a força das gotas da chuva, fornece através do escoamento pelo tronco, o auto abastecimento de água no solo e a lixiviação de alguns íons (JOHNSSON, 1990).

Entre os totais médios anuais de precipitação efetiva, cerca de $56 \%$ atingiu o solo com passagem direta, aproximadamente $40 \%$ foi interceptada pelo dossel da planta e atingiu a superfície por escoamento de haste ou gotejamento das folhas e os $4 \%$ restantes correspondem a parte interceptada, armazenada pelo dossel e em seguida evaporada. 

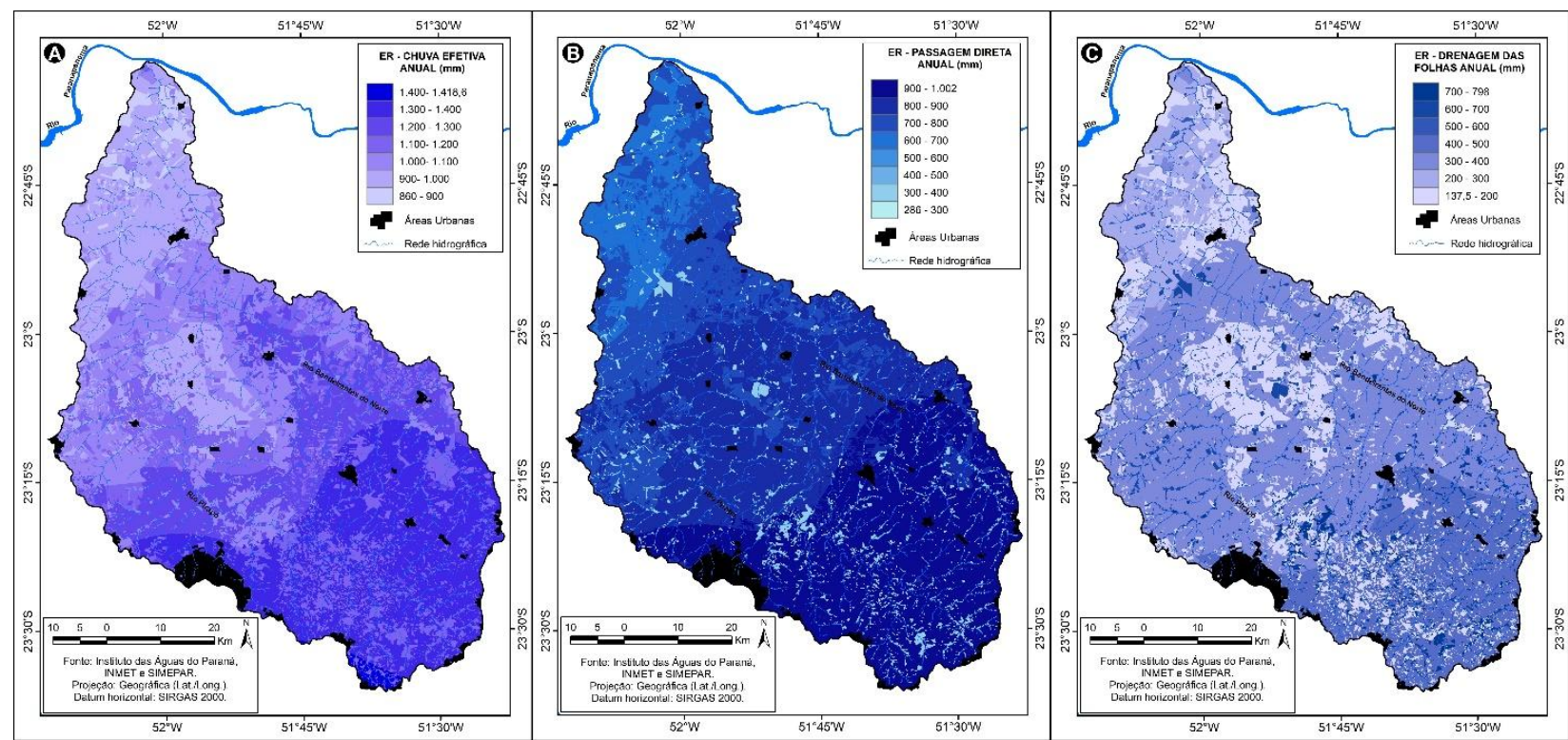

Figura 3: A) Chuva efetiva anual; B) Chuva efetiva anual por passagem direta; C) Chuva efetiva anual por drenagem das folhas.

O valor médio anual da precipitação efetiva com passagem direta corresponde a $654 \mathrm{~mm}$, com intervalos entre 1.002 e $226 \mathrm{~mm}$ (Figura 3 - B), concentrando-se mais no setor sul da bacia, onde ocorrem os valores mais expressivos de precipitação e ocupado com culturas temporárias, que são mais vulneráveis a ocorrência de escoamento em superfície.

A precipitação interceptada pelo dossel da planta e que atinge a superfície por escoamento de haste ou gotejamento das folhas apresentou um valor médio anual de $467 \mathrm{~mm}$, distribuídos entre os intervalos de 137 a $798 \mathrm{~mm}$ (Figura 3 - C). É mais representativa nas áreas com ocorrência de floresta ou silvicultura, onde prevalece a infiltração da água no solo.

Os valores de energia cinética total são mais significativos na porção centro-sul da bacia, com valores superiores a $2.500 \mathrm{MJ}^{-h a^{-1}}$.ano ${ }^{-1}$, comportamento similar ao encontrado por Terassi et al. (2016), embora os valores obtidos sejam menores, com intervalos entre 1.773 a 2.998 MJ.ha $^{-1} \cdot$ ano $^{-1}$ (Figura 4 - A). Os valores mais baixos encontrados em relação a outros trabalhos que contemplaram a região da bacia (WALTRIK et al., 2012 e TERASSI et al., 2016) pode ser explicado, em parte, pela diferença entre a aplicação dos valores de precipitação, uma vez que, nesses trabalhos foram utilizados apenas valores totais médios de pluviosidade.
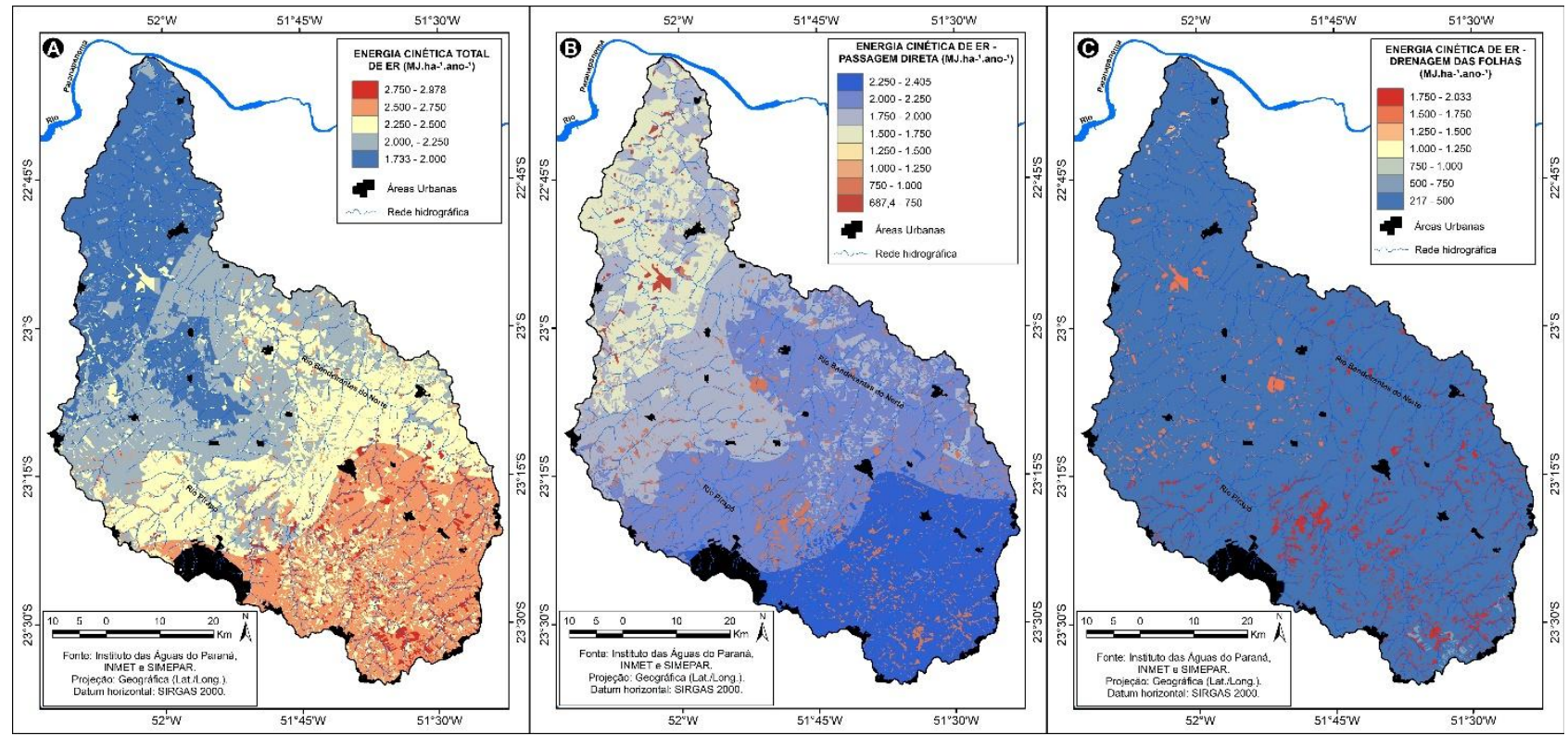

Figura 4: A) Energia cinética anual; B) Energia cinética anual por passagem direta; C) Energia cinética anual por drenagem das folhas. 
Os totais de energia cinética com passagem direta são mais representativos sobre as áreas da bacia com cultivos temporárias (soja, milho, trigo e cana-de-açúcar), representado cerca de $66 \%$ dos valores acima da média da bacia, que é de $1.546 \mathrm{MJ}^{-} \mathrm{ha}^{-1}$.ano ${ }^{-1}$. Os valores representativos ficaram entre 687 e $2.405 \mathrm{MJ}^{-h^{-}}$ ${ }^{1}$. ano $^{-1}$ (Figura 4 - B).

A distribuição dos valores de energia cinética interceptada pelo dossel das plantas que atingiu o solo por escoamento de haste ou gotejamento das folhas apresentou um padrão mais homogêneo, com aproximadamente $90 \%$ da bacia concentrando valores abaixo de $500 \mathrm{MJ}^{-h^{-1}}$. ano ${ }^{-1}$. Os maiores valores, como era de se esperar, são encontrados onde ocorrem a cobertura vegetal densa/mata, com totais de $2.033 \mathrm{MJ}^{-\mathrm{ha}^{-}}$ ${ }^{1}$. ano ${ }^{-1}$ (Figura 4 - C).

O escoamento superficial somente se inicia quando a intensidade de chuva é superior à taxa de infiltração instantânea do solo (PINESE JUNIOR et al., 2008), favorecendo o transporte dos materiais de solo que foram desagregados pelo impacto direto/indireto da chuva. Além das condições climáticas, como o volume precipitado e a intensidade, têm-se outros fatores que ajudam a romper o equilíbrio natural do solo, como a declividade do terreno, a capacidade de infiltração da água e a sua resistência à ação erosiva da água, a distância percorrida pelo escoamento superficial, a rugosidade superficial do terreno, o volume de cobertura do solo dada a ocorrência da chuva e o uso e manejo do solo (PRUSKI, 2008).

De modo geral, o escoamento superficial não apresentou valores tão elevados, sendo predominantemente identificados valores médios entre 10 e $50 \mathrm{~mm} \cdot \mathrm{ha}^{-1} \cdot \mathrm{ano}^{-1}$, que ocorrem em cerca de $65 \%$ da área da bacia (Figura 5 - A), associados a presença de solos mais profundos e bem drenados, como os Latossolos e Nitossolos sobre cultivo temporários.

Os valores mais elevados de escoamento superficial foram identificados no setor sul da bacia, com valores acima de $100 \mathrm{~mm} \cdot \mathrm{ha}^{-1} \cdot$ ano $^{-1}$ (Figura 5 - A), que estão relacionados a presença de declividades mais acentuados em solos rasos, como o Neossolos Litólicos/Regolíticos, que pode ser agravado considerando o uso por cultivos temporários, facilitando a formação do escoamento superficial e a ocorrência de processos erosivos (SARTORI et al., 2005).

Os menores valores são encontrados no setor norte da bacia, inferiores a 10 mm.ha ${ }^{-1} \cdot$ ano $^{-1}$ (Figura 5 - A), onde predominam solos de textura média/arenosa, como os Argissolos. Os solos arenosos em termos da relação infiltração e escoamento, pode ser problemático se o horizonte superficial do solo for arenoso, e o horizonte logo abaixo for mal drenado.

Segundo Pinese Junior et al. (2008), o escoamento superficial é inversamente proporcional à cobertura vegetal, indicando que esta é uma proteção eficaz para a contenção do fluxo superficial, por oferecer uma barreira física contra o aumento da energia cinética do escoamento. Além da ação destrutiva que promove no solo, o escoamento superficial constitui o principal meio de contaminação dos mananciais de água superficial devido ao transporte de sedimentos e produtos químicos.

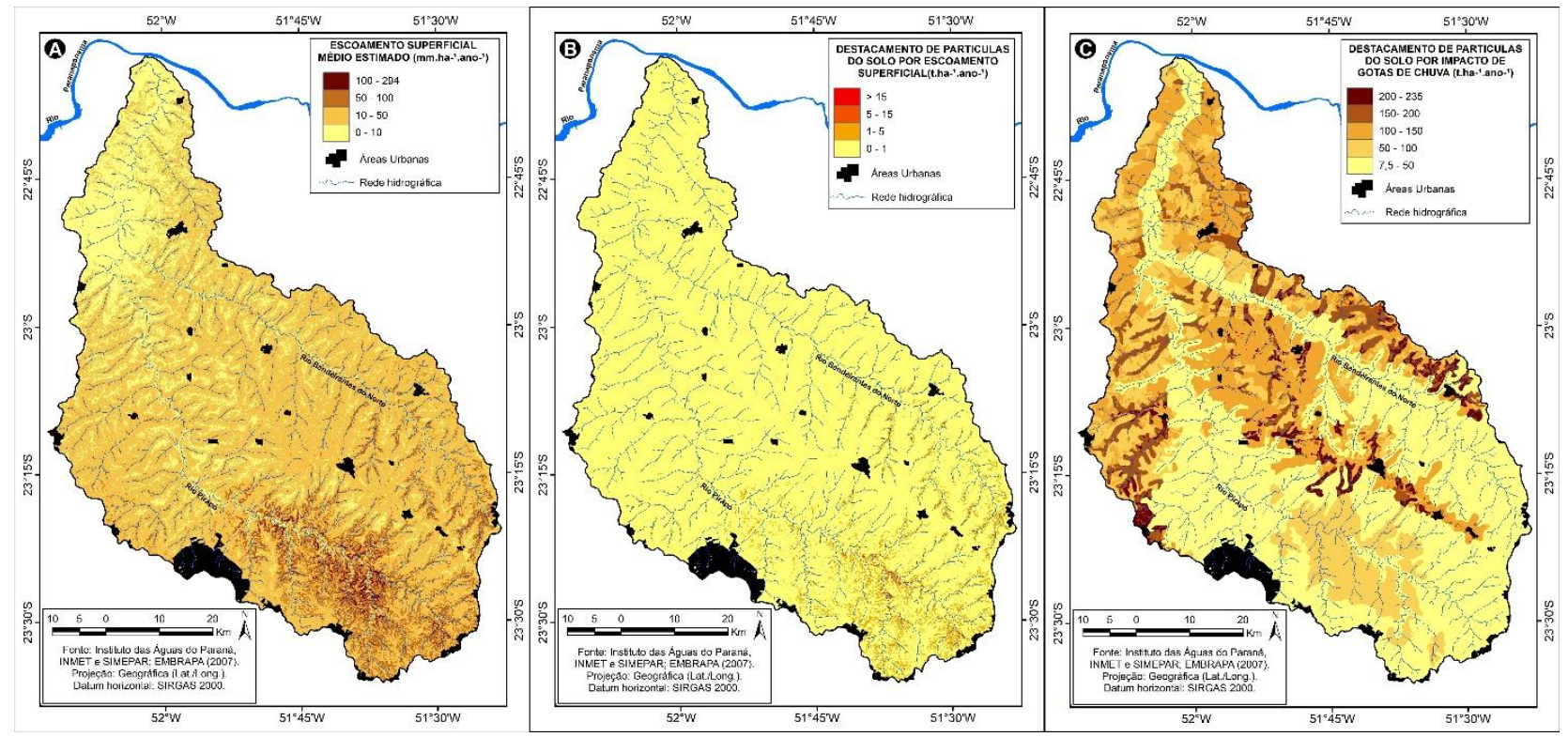

Figura 5: A) Escoamento superficial médio estimado; B) Destacamento de partículas de solo por escoamento superficial; C) Destacamento de partículas de solo por impacto de gotas de chuva. 
Os maiores valores, próximos a 15 t.ha $^{-1} \cdot$ ano $^{-1}$, relacionados a esse processo são identificados no setor sul da bacia, em áreas com presença de solos rasos (Neossolos Litólicos/Regolíticos) e declividades acentuadas, superiores a 16\%, próxima as calhas dos rios (Figura 5 - B).

O destacamento de partículas do solo por impactos das gotas da chuva apresentou um maior poder erosivo quando relacionado ao destacamento de partículas dos solos por escoamento superficial, segundo Hudson (1965) isso ocorre devido ao impacto da gota de chuva que tem energia cinética 256 vezes maior que a energia do escoamento superficial.

Os valores relacionados a esse processo são mais significativos, principalmente no setor centro-norte da bacia, com totais superando as 100 t.ha $^{-1} \cdot$ ano $^{-1}$ (Figura 5 - B), onde são encontrados solos de textura média e arenosa. Esse enquadramento vai de encontro com as análises realizadas por Marcatto (2016), que apontaram uma maior suscetibilidade a formação de processos erosivos nos solos com textura média e arenosa quando comparados aos solos com textura argilosa ou muito argilosa, isso para diferentes tipos de usos na bacia.

A taxa de destacamento anual apresentou valores totais similares aos encontrados pelo destacamento de partículas dos solos por impactos das gotas da chuva (Figura 5 - C e $\mathbf{6}$ - A), que é função desse processo com destacamento de partículas dos solos por escoamento superficial.

Os valores anuais da taxa de destacamento de partículas assumem maior importância por representar o total de sedimentos disponíveis por tipo de solo e uso do solo para transporte, podendo ser depositado em setores mais baixos do relevo e nos leitos dos rios.

A capacidade de transporte de sedimentos por escoamento superficial apresentou valores relativamente baixos, com média de aproximadamente 10 t.ha ${ }^{-1} \cdot$ ano $^{-1}$, com maiores valores concentrados na setor sul e redução gradativa no sentido norte da bacia (Figura 6 - B), influenciada principalmente pela redução nos valores de precipitação.

Diante disso, foram relacionados os valores de capacidade de transporte de sedimento e taxa anual de desprendimento para estabelecer a vulnerabilidade à contaminação das águas superficiais. Essa classificação segue o princípio de que em áreas intensamente agrícolas a probabilidade de os sedimentos serem transportados são grandes, devido as alterações sistemáticas promovidas nesse ambiente, além disso, tem-se a excessiva utilização de agroquímicos ao longo dos ciclos produtivos.

Desse modo, dentro do conceito de vulnerabilidade à contaminação das águas superficiais, os agroquímicos serviram como índice potencializador dentro da relação capacidade de transporte e taxa anual de destacamento de partículas do solo.

$\mathrm{Na}$ bacia foram encontradas 5 classes de vulnerabilidade, a maior porcentagem esteve ligada à vulnerabilidade média, que corresponde cerca de $63 \%$ da área da bacia, seguida pelas classes baixa e alta, com valores próximos a $23 \%$ e $7 \%$, respectivamente (Tabela 6).


Figura 6: A) Taxa de desprendimento anual; B) Capacidade de transporte de sedimentos por escoamento superficial; C) Vulnerabilidade à contaminação das águas superficiais. 
Tabela 6: Áreas das classes de vulnerabilidade à contaminação das águas superficiais (face pesticida) na bacia do rio Pirapó - PR.

\begin{tabular}{|c|c|c|c|}
\hline \multirow[b]{2}{*}{ Intervalos } & \multirow[b]{2}{*}{ Classes } & \multicolumn{2}{|c|}{ Área* } \\
\hline & & $\left(\mathbf{K m}^{2}\right)$ & $(\%)$ \\
\hline $4,8-6,0$ & Muito Elevada & 38,72 & 0,78 \\
\hline $3,6-4,8$ & Elevada & 353,87 & 7,11 \\
\hline $2,4-3,6$ & Média & 3133,15 & 62,98 \\
\hline $1,2-2,4$ & Baixa & 1142,33 & 22,96 \\
\hline $0,0-1,2$ & Muito Baixa & 306,71 & 6,17 \\
\hline
\end{tabular}

Fonte: Autores. *Não foram consideradas as áreas urbanas no cálculo.

Nos setores da bacia onde são identificadas a vulnerabilidade muito elevada e elevada a contaminação das águas superficiais (Figura 6 - C), são predominantemente ocupados por cultivos temporários, associados com valores mais intenso de escoamento superficial sobre áreas de declividades mais acentuadas. A diferenciação entre as classes está relacionada aos tipos de solos, onde são rasos (Neossolos Litólicos e Regolíticos) a possibilidade de ocorrer escoamento é maior comparada com outros solos e, assim, uma maior capacidade de transporte de contaminantes.

Para os setores com média vulnerabilidade à contaminação das águas superficiais (Figura 6 - C), sua classificação, está relacionada a ocorrência de solos profundos e resistentes ao desprendimento de partículas, com valores mais baixos de escoamento superficial influenciados pela redução na intensidade da precipitação, além de estar sobre áreas de declividade moderadas, com predomino de classes ente 8 e $15 \%$. Mesmo sobre condições físicas mais atenuantes, os usos predominantes estão relacionados aos cultivos temporários que apresentam altos valores de usos de agroquímicos que intensificam os riscos nesses setores da bacia.

Nos setores classificados como baixa e muito baixa vulnerabilidade contaminação das águas superficiais, sua classificação está associada a presença de áreas florestadas e pastagens, usos com pouca ou nenhuma utilização de agroquímicos, reduzindo os riscos, mesmo ocorrendo em áreas com taxas de escoamento e transporte de partículas elevados.

\section{Considerações finais}

Com a aplicação do modelo voltado a estabelecer as vulnerabilidades à contaminação das águas superficiais foi possível identificar cinco classes de vulnerabilidade, a maior porcentagem esteve ligada à vulnerabilidade média, correspondente a cerca de $63 \%$ da área da bacia. Os setores da bacia onde são identificadas a vulnerabilidade muito elevada e elevada, são predominantemente ocupados por cultivos temporários, associados com valores mais intenso de escoamento superficial sobre áreas de declividades mais acentuadas.

Assim, considera-se que o estudo possa contribuir no desenvolvimento de novas ações e estratégicas para a proteção das águas superficiais da bacia, priorizando as áreas mais críticas para o monitoramento de suas águas.

Agradecimentos

A Coordenação de Aperfeiçoamento de Pessoal de Nível Superior (CAPES) pelo fomento durante o período de realização do trabalho.

\section{Referências}

AGNINDHIRA, G. P. Finding the Optimal Unsaturated Zone Storage Change Estimation Method to Improve Moisture Accounting in Vietnam: A case study of the $\mathrm{Ca}$ and the Downstream Red River Basin in Vietnam. 2016. 70f. Dissertação (Mestrado em Ciências), Delft University of Technology, Netherlands, 2016.

ALVES, E. C.; SILVA, C. F.; COSSICH, E. S.; TAVARES, C. R. G.; FILHO, E. E. S.; CARNIEL, A. Avaliação da qualidade da água da bacia do rio Pirapó - Maringá, Estado do Paraná, por meio de parâmetros físicos, químicos e microbiológicos. Acta Scientiarum Technology, v. 30, n. 1, p. 39-48. 2008. DOI: https://doi.org/10.4025/actascitechnol.v30i1.3199. 
ALVES FILHO, J. P. Uso de agrotóxicos no Brasil: controle social e interesses corporativos. São Paulo: Annablume, 2002.

BERTONI, J.; LOMBARDI NETO, F. Conservação do solo. $8^{\text {a }}$ ed. São Paulo: Ícone, 2012.

BOUYOUCOS, G. J. The Clay ratio as a criterion of susceptibility of soils toerosion. Journal of the American Society of Agronomy, 27: 738-741. 1935.

BRANDT, C.J. Simulation of the size distribution and erosivity of raindrops and throughfall. 1990.

DEVIA, G.K.; GANASRI, B.; DWARAKISH, G. A Review on Hydrological Models. Aquatic Procedia, 4, 1001-1007. 2015. DOI: https://doi.org/10.1016/j.aqpro.2015.02.126

EMBRAPA (Empresa Brasileira de Pesquisa Agropecuária). Mapa de Solos do Estado do Paraná. Rio de Janeiro, Centro Nacional de Pesquisa de Solos: EMBRAPA: Solos e Florestas, 2007. 95p.

FREIRE, R. Monitoramento da qualidade da água da bacia hidrográfica do ribeirão Maringá. 2010. 175f. Dissertação (Mestrado em Engenharia Química), Universidade Estadual de Maringá, Maringá, 2010.

FREIRE, R. BONIFÁCIO, C. M.; SCHNEIDER, R. M. Determinação de cargas poluidoras em cursos d'água de médio porte. In: BENINI, S. M.; DIAS, L. S.; BENINI, E. M. Avaliações ambientais em bacias hidrográficas. Tupã: ANAP, p. 124 - 144. 2014.

EFTHIMIOU, N., LYKOUDI, E.; KARAVITIS, C. Comparative analysis of sediment yield estimations using different empirical soil erosion models. Hydrological Sciences Journal, 62(16), 2674-2694. 2017. DOI: https://doi.org/10.1080/02626667.2017.1404068.

EFTHIMIOU, N. Development and testing of the Revised Morgan-Morgan-Finney (RMMF) soil erosion model under different pedological datasets. Hydrological Sciences Journal, 64(9), 1095-1116, 2019. DOI: https://doi.org/10.1080/02626667.2019.1623896.

GOVERS, G. Empirical relationships for the transport capacity of overland flow. Erosion, Transport and Deposition Processes. Jerusalem, IAHS Publication 189: 45-63. 1990.

HUDSON, N.W. The influence of rainfall on the mechanics of soil erosion with particular reference to Southern Rhodesia. 1965. (Msc Thesis), University of Cape Town, Cape Town. 1965.

JOHNSSON, R. G. The interception, throughfall and stemflow in a Forest in Highland Scotland and the comparison with other upland forests in the U. K. Journal of Hidrology, Amsterdam, v. 118, p. 281-287, 1990.

LILHARE, R.; GARG, V.; NIKAM, B. Application of GIS-Coupled Modified MMF Model to Estimate Sediment Yield on aWatershed Scale. Journal of Hydrologic Engineering, 20(6), 2015. DOI: 10.1061/(ASCE)HE.1943-5584.0001063.

LUGER, C.E.M.; MICHAILOVSKY, C. BASTIAANSSEN, W.; BOGAARD, T. Redesigning the MorganMorgan-Finney Soil Erosion Model for Global High-Resolution Application: By using remote sensing data as the main source of input and coupling runoff calculation to the IHE hydrological model, WaterPix. Water Accounting, IHE Delft Institute for Water Education, Westvest- Netherlands. 2018. 28p.

MARCATTO, F. S. O efeito do uso e manejo nas propriedades físicas e hídricas dos solos da bacia hidrográfica do rio Pirapó - PR. 2016. 103f. Dissertação (Mestrado em Geografia) - Universidade Estadual de Maringá - UEM. 2016.

MARCATTO, F. S.; SILVEIRA, H. Relação entre as propriedades físicas e hídricas e os tipos de uso da terra em Neossolos Regolíticos da bacia hidrográfica do rio Pirapó - PR. Caderno de Geografia. v.27, n.50, 2017. DOI: https://doi.org/10.5752/p.2318-2962.2017v27n50p427. 
MEYER, L. D.; WISCHMEIER, W. H. Mathematical simulation of the process of soil erosion by water', Transactions of the ASAE. American Society of Agricultural and Biological Engineers, 12(6), pp. 754 758. 1969.

MINEROPAR. Atlas Geológico do Estado do Paraná. Curitiba. 2001. 125p. CD ROM...

MINGOTI, R., SPADOTTO, C. A.; MORAES, D. A. C. Suscetibilidade à contaminação da água subterrânea em função de propriedades dos solos no Cerrado brasileiro. Pesquisa Agropecuária Brasileira, Brasília, v.51, n.9, p.1252-1260, 2016. DOI: https://doi.org/10.1590/s0100-204x2016000900025.

MORGAN, R.P.C. Soil Erosion and Conservation. Longman. 1995. 198 pp.

MORGAN, R.P.C. A simple approach to soil loss prediction: a revised Morgan-Morgan-Finney model. Catena, 44, pp. 305-322. 2001.

MORGAN, R.P.C., MORGAN, D.D.V.; FINNEY, H.J. A predictive model for the assessment of soil erosion risk. Journal of Agric, Eng. Res., 30, pp. 245-253. 1984.

MORGAN, R.P.C., QUINTON, J.N., RICKSON, R.J. EUROSEM: A User Guide. Silsoe College, Cranfield University. 1993.

NITSCHE, P. R.; CARAMORI, P. H; RICCE, W. S.; PINTO, L. F. D.. Atlas Climático do Estado do Paraná. Londrina, PR: IAPAR, 2019.

NÓBREGA, M.T.; SERRA, E.; SILVEIRA, H.; TERASSI, P.M.B.; BONIFÁCIO, C.M. Landscape structure in the Pirapó, Paranapanema 3 and 4 Hydrographic Unit, in the state of Paraná. Brazilian Journal of Biology, v.75, n.4, 2015. DOI: https://doi.org/10.1590/1519-6984.00913suppl.

OLIVEIRA, A.M.M., PINTO, S.A.F. E LOMBARDI NETO, F. Caracterização de indicadores da erosão do solo em bacias hidrográficas com o suporte de geotecnologias e modelo predictivo. Estudos Geográficos, vol. 5, no. 1, pp. 63-86. 2007.

OLLI, G.; DARRACQ, A.; DESTOUNI, G. Field study of phosphorous transport and retention in drainage $\begin{array}{llllllllll}\text { reaches. Journal of Hydrology, } & \text { v. } 365, & \text { n. } & 1, & \text { p. } & 46-55, & 2009 . & \text { DOI: }\end{array}$ https://doi.org/10.1016/j.jhydrol.2008.11.039

PINESE JUNIOR, J. F.; CRUZ, L. M.; RODRIGUES, S. C. Monitoramento de erosão laminar em diferentes usos da terra, Uberlândia - MG. Sociedade \& Natureza (Online), Uberlândia, v. 20, n. 2, p. 157-175, 2008.

PINTO, H. S.; ALFONSI, R. R. Estimativa das temperaturas médias, máximas e mínimas mensais no Estado do Paraná, em função de altitude e latitude. Caderno de Ciências da Terra, v.52. 1974. 28p.

PRUSKI, F., BRANDÃO, V. S. E SILVA, D. D. Escoamento Superficial. Editora da UFV, 4ª ed, 2008.

RAUWS, G., GOVERS, G. Hydraulic and soil mechanical aspects of rill generation on agricultural soils. Journal of Soil Science. 39, 111-124. 1988.

RUFINO, R. L.; BISCAIA, R. C. M.; MERTEN, G. H. Determinação do potencial erosivo da chuva do estado do Paraná, através de pluviometria: terceira aproximação. Revista Brasileira de Ciência do Solo, v. 17, n.1, p. 439-444,1993.

SARTORI, A.; LOMBARDI NETO, F.; GENOVEZ, A. M. Classificação hidrológica de solos brasileiros para a estimativa da chuva excedente com o método do serviço de conservação do solo dos Estados Unidos. Parte 1: Classificação. Revista Brasileira de Recursos Hídricos, 2005. DOI: 10.21168/rbrh.v10n4.p19-29

SCORZA JUNIOR, R. P.; NÉVOLA, F. A.; AYELO, V. S.; Avaliação da contaminação hídrica por agrotóxico. Boletim de pesquisa e desenvolvimento. Dourados: EMBRAPA Agropecuária Oeste, 2010. 
SESA (Secretaria do Estado da Saúde do Paraná). Plano de Vigilância e Atenção à Saúde de Populações Expostas aos Agrotóxicos do Estado do Paraná 2017 a 2019. Curitiba. 2018. Disponível em:< http://www.saude.pr.gov.br/arquivos/File/PlanoAgrotoxic30_05_18.pdf $>$. Acesso em: 08 de setembro de 2018

SIAGRO (Agência de Defesa Agropecuária do Paraná - ADAPAR). Banco de Dados de Agrotóxicos. 2018. Disponível em: < http://www.adapar.pr.gov.br/>. Acesso em: 16 março 2018.

SILVA, A.M.; SCHULZ, H.E.; CAMARGO, P.B. Erosão e Hidrossedimentologia em Bacias Hidrográficas. 2. ed. São Carlos: RIMA, 2007. 153p.

SILVA, D. F.; SOUSA, F. A. S.; KAYANO, M. T. Escalas temporais da variabilidade pluviométrica na bacia hidrográfica do rio Mundaú. Revista Brasileira de Meteorologia, São Paulo, v. 25, n.3, p. 147-155, 2010. DOI: https://doi.org/10.1590/S0102-77862010000300004.

SILVA, L. H. B. R. Monitoramento da qualidade e análise sedimentar da bacia hidrográfica do rio Pirapó influência da vazão. 2017. 105f. Dissertação (Mestrado em Engenharia Química), Universidade Estadual de Maringá, Maringá. 2017.

SPADOTTO, C.A.; HOLLER, W.A.; MARTINHO, P.R.R.; FOIS, N.S.; MORAES, D.A. DE C.; DALTIO, J.; TRAJANO, S.R. da S. Interfaces de modelos ambientais e sistemas de informação geográfica para a gestão territorial da contaminação de recursos hídricos. Campinas: Embrapa Gestão Territorial, 2012. $37 \mathrm{p}$.

SPERLING, M. V. Estudos e modelagem da qualidade da água de rios. Editora: UFMG, v. 7., 2007. $473 p$.

STEENHUIS, T. S., WINCHELL, M., ROSSING, J., ZOLLWEG, J. A.; WALTER, M. F. SCS Runoff Equation Revisited for Variable-Source Runoff Areas. Journal of Irrigation and Drainage Engineering, 121(3), 234-238. 1995. DOI: 10.1061/(ASCE)0733-9437(1995)121:3(234)

SUTTON, A. J.; FISHER, T. R. Historical changes in water quality at german Branch in choptank river basin. Water, Air and Soil Pollution, v. 199, n. 4, pp. 353-369, 2009.

TESFAHUNEGN, G. B., TAMENE, L.; VLEK, P. L. Soil erosion prediction using Morgan-Morgan-Finney model in a GIS environment in Northern Ethiopia catchment. Applied and Environmental Soil Science, 2014, 15p. DOI: https://doi.org/10.1155/2014/468751

TERASSI, P. M. B.; SILVEIRA, HÉLIO; GRAÇA, C. H. Regiões pluviométricas homogêneas e a erosividade das chuvas na unidade hidrográfica Pirapó, Paranapanema III e IV - Paraná. Caderno de Geografia, v. 26, p. 507-521, 2016. DOI: https://doi.org/10.5752/P.2318-2962.2016v26n46p507

TOMAZONI, J.C.; GUIMARÃES, E. A sistematização dos fatores da EUPS em SIG para quantificação da erosão laminar na bacia do rio Jirau. Revista Brasileira de Cartografia, no. 57, pp. 235-244. 2005.

TURC, L. Water balance of soils: relationship between precipitation, evapotranspiration and runoff. Ann Agron, 1954.

VÁZQUEZ-FERNÁNDEZ, G.A., FORMAGGIO, A.R., EPIPHANIO, J.C.N.; GLERIANI, J.M. Determinação de sequências culturais em microbacia hidrográfica para caracterização do Fator C da EUPS, utilizando fotografia aérea. In: Anais... VIII Simpósio Brasileiro de Sensoriamento Remoto, 1996, Salvador. São José dos Campos: INPE, pp. 63-67. 1996.

VIGIAK, O., OKOBA, B. O., STERK, G. GROENENBERG, S. Modelling catchment-scale erosion patterns in the East African Highlands. Earth Surface Processes and Landforms, 30: 183 -196. 2005. DOI: https://doi.org/10.1002/esp.1174

WALTRICK, P. C.; MACHADO, M. A. M.; DIECKOW, J.; OLIVEIRA, D. Estimativas da erosividade de chuvas no estado do Paraná pelo método da pluviometria: Atualização com dados de 1986 a 2008. Revista 
Brasileira de Ciência do Solo, Viçosa, v.39, n.1, p.256-267, 2015. DOI: https://doi.org/10.1590/01000683rbcs20150147

WISCHMEIER, W.H.; SMITH, D.D. Predicting rainfall erosion losses: a guide to conservation planning. USDA Agricultural Handbook, vol. 537. 1978.

(1) (9) (2) 